The Journal of Trauma: Injury, Infection, and Critical Care

Issue: Volume 43(6), December 1997, pp 975-976

Copyright: @ Williams \& Wilkins 1997. All Rights Reserved.

Publication Type: [Case Reports]

ISSN: 0022-5282

Accession: 00005373-199712000-00021

[Case Reports]

\title{
Ventral Tension Pneumothorax
}

Vermeulen, Erik G. J. MD; Teng, H. T. MD; Boxma, H. MD, PhD

\section{Author Information}

From the Department of General Surgery (E.G.J.V., H.B.), and Department of Radiology (H.T.T.),

Zuiderziekenhuis, Rotterdam, The Netherlands.

Address for reprints: Dr. H. Boxma, Department of Surgery, Zuiderziekenhuis, Groene Hilledijk 315, 3075

EA Rotterdam, The Netherlands.

Chest injuries are the cause of death in $25 \%$ of trauma fatalities and a major contributing factor in an additional 50\%. Pneumothorax, the second most common chest injury, may often be overlooked, and this oversight may lead to deterioration, significant complications, and/or death. This case report describes such a failure because of a rare abnormal localization (ventral) of the pneumothorax and the shortcoming of a supine anterior-posterior chest x-ray film in the trauma room.

\section{CASE REPORT}

A 56-year-old patient was admitted to the first aid department after he had jumped off the roof of a three-story building in a suicide attempt. The patient was awake and complained about pains in both forearms, the right ankle, and the lower back.

Physical examination, with the patient lying on a first aid stretcher, revealed a noninvasive blood pressure of $110 / 65$ with a pulse rate of 88 . Head and neck showed no abnormalities and auscultation of the lungs was normal. There was no shortness of breath. Abdomen and extremities showed no abnormalities, except signs of closed forearm fractures on both sides.

Initial x-ray films made of the chest (Figure 1) and the pelvis showed no abnormalities. Further radiography of the patient showed unstable fractures of lumbar vertebrae 1 and 3 and distal dislocated forearm fractures on both sides. During radiography the systolic blood pressure of the patient dropped to $70 \mathrm{~mm} \mathrm{Hg}$, and rapid infusion was started under the suspicion of hypovolemia. Blood pressure stabilized, but rapid infusion had to be maintained. Repeated physical examination gave no additional information. Repeated chest x-ray film again showed no signs of pneumo- or hematothorax or mediastinal widening, and due to the low suspicion of an intrathoracic pathologic condition, an empiric chest tube was not placed. Ultrasonography of the abdomen was performed, but due to intestinal gases only the lower part could be visualized. This image showed no signs of bleeding. Ultrasonography of the chest was not performed. 


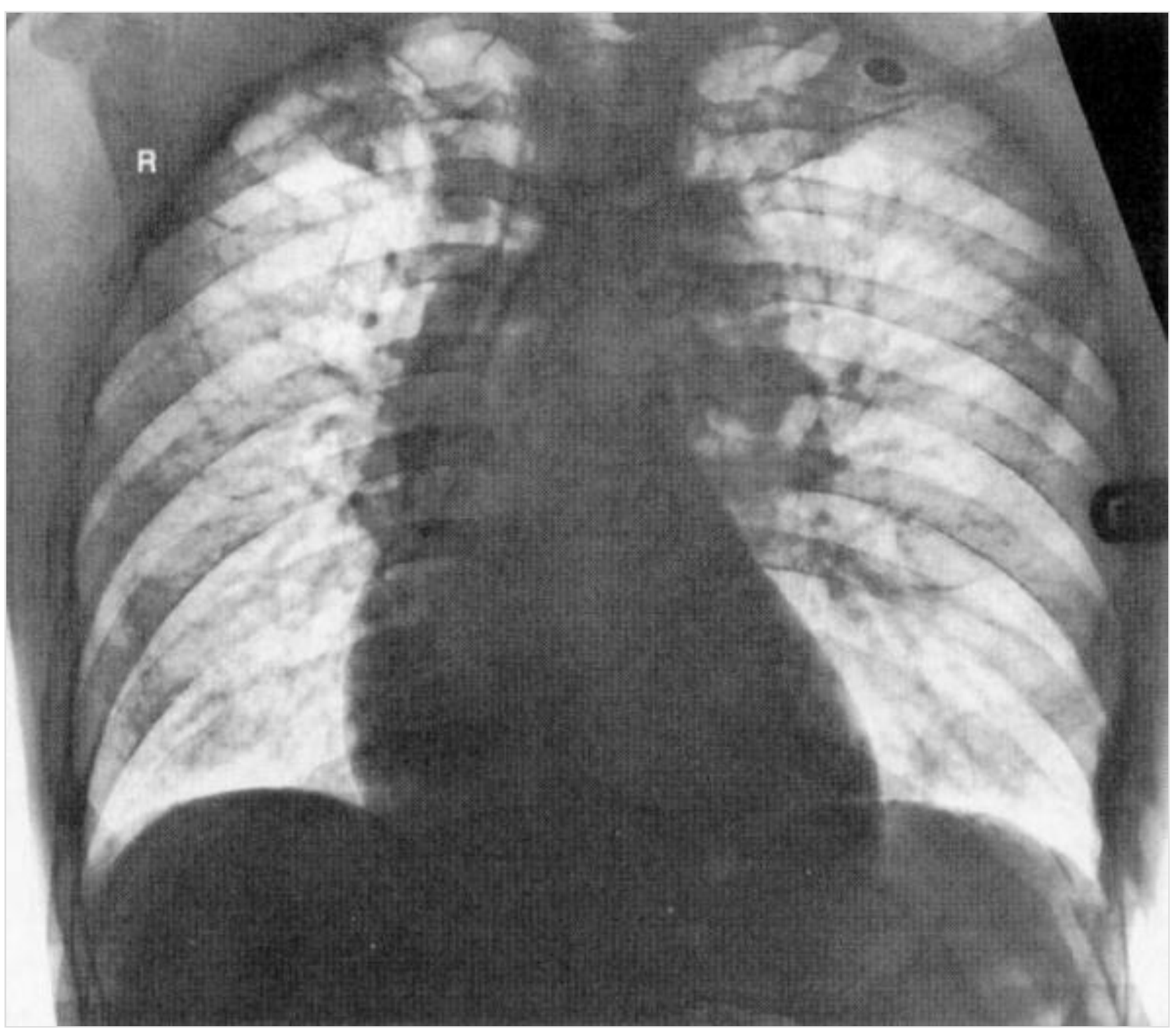

Figure 1. Supine chest x-ray film on admission at the first aid department showing no abnormalities.

The patient remained infusion dependent, and it was decided to perform a computed tomographic (CT) scan to evaluate the possibility of intra-abdominal bleeding, intrathoracic bleeding, or both, and to classify the lumbar spine injury. Surprisingly on CT scan the real reason for the clinical unstable condition proved to be a ventral pneumothorax on the left side (Figure 2) and a chest tube was placed without delay. 


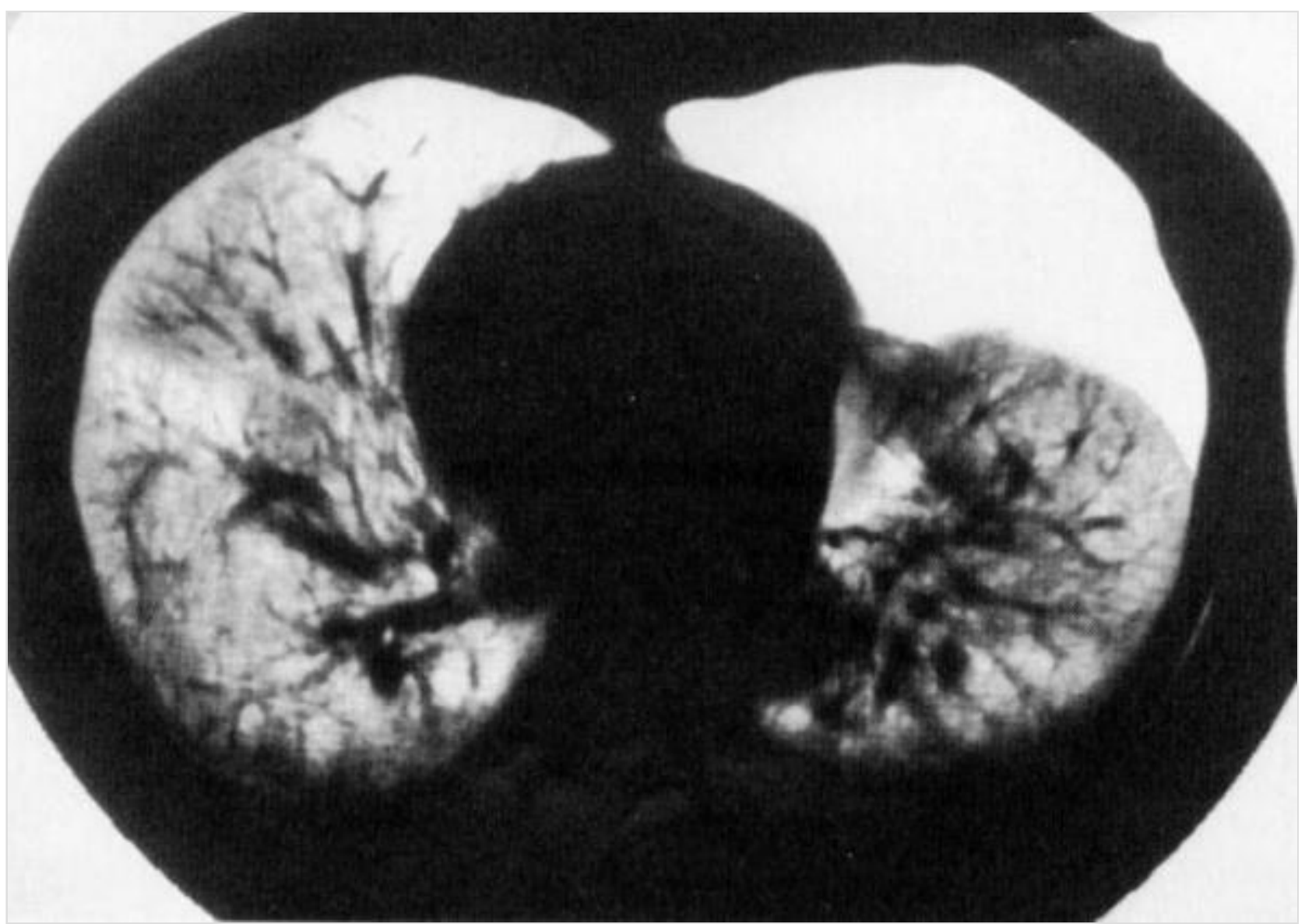

Figure 2. A CT scan of the chest showing clearly the tension pneumothorax as a white area on the left ventral side.

\section{DISCUSSION}

The resuscitation of trauma patients in the first aid department is getting more and more attention every year. It becomes clear to most surgeons that the resuscitation in the first (golden) hour is essential for the possible recovery of the patient. Special training such as the Advanced Trauma Life Support (ATLS) is recommended for every doctor who works in the first aid department and should be obligatory to those specialized in trauma care. One feature of every training program is the renewed learning of a good and systematic physical examination of the patient. With this examination most of the abnormalities can be diagnosed or at least suspected. The ABC (airway-breathing-circulation) protocol should always be followed. After the physical examination (and interpretation of this examination), the radiologist can confirm the diagnosis either by $\mathrm{x}$-ray film, ultrasound, or more sophisticated examinations.

Hemodynamic instability in the trauma patient will nearly always have a hypovolemic cause, but beware of tension pneumothorax, cardiac tamponade, myocardial contusion, or lung embolus. Intravascular volume depletion may occur due to bleeding in the thoracal cavity, abdominal cavity, or both, or in the extremities due to fractures. Examination should point out the causative localization(s), and these should be treated promptly. But sometimes physical and radiologic examinations do not reveal any abnormality in the hemodynamically unstable patient. In those cases one should remind oneself that the examinations do not have a $100 \%$ sensitivity. 
This insensitivity was perfectly demonstrated in the here-reported case. Apart from the possible failure in this case to detect the pneumothorax by physical examination due to its circumscript localization (partially anterior to the heart and the left lung), the anterior-posterior chest x-ray film (Figure 1) failed to detect the pneumothorax on the left side. An upright anterior-posterior chest $\mathrm{x}$-ray film is more sensitive for a pneumothorax than is a supine one, but due to the spine fractures in this patient, only supine $x$-ray films could be made. A CT scan revealed the pneumothorax. If on the other hand the possibility of a ventral tension pneumothorax was considered in the first aid department, a lateral chest x-ray film could have revealed the true problem. In 14 to $29 \%$ of the cases a lateral chest $x$-ray film can be helpful in either recognizing or localizing a pneumothorax. [1,2] No data are given regarding the clinical relevance of this pneumothorax.

The hemodynamic instability of the patient and the stabilization of this condition after chest tube placement confirm that the patient had a tension pneumothorax (pressure in the pleural space exceeds atmospheric pressure). It is known that severe cardiovascular and pulmonary compromise may result from a small volume of pleural air under tension. $[3,4]$ The degree of lung collapse depends on pleural adhesions, lung stiffness, and compressibility, and mediastinal shift depends on the ability of the contralateral lung to decrease in volume. [3] Thus the only radiographic evidence of a tension pneumothorax may be slight flattening of the cardiac border and ipsilateral contour change or depression of the diaphragm. The other possible signs of a pneumothorax on a supine chest radiograph are deep sulcus sign, sharp hemidiaphragmatic border, sharp mediastinal contours, hyperlucency above liver and spleen, hyperlucency of entire hemithorax, and pneumomediastinum. [5-7] Chest ultrasonography is another possibility to reveal pneumothorax, but it is highly dependent of the experience of the radiologist.

In conclusion we state that a lateral chest radiography in unstable trauma patients without any other obvious cause can provide helpful information in addition to the AP view but should only be used in selected cases of clinical importance as is here in the case of a ventral tension pneumothorax.

\section{REFERENCES}

1. Glazer HS, Anderson DJ, Wilson BS, et al. Pneumothorax: appearance on lateral chest radiographs. Radiology. 1989;173:707. Full Text @ EUR | Bibliographic Links | [Context Link]

2. Morgan RA, Owens CM, Collins CD, et al. Detection of pneumothorax with lateral shoot-through digital radiography. Clin Radiol. 1993;48:249-252. Full Text @ EUR | Full Text | Bibliographic Links [Context Link]

3. Gobien RP, Reines HD, Schabel SI. Localized pneumothorax: unrecognized form of barotrauma in adult respiratory distress syndrome. Radiology. 1982;142:15-19. Full Text @ EUR | Bibliographic Links [Context Link]

4. Tocino IM, Miller MH, Fairfax WR. Distribution of pneumothorax in the supine and semirecumbent critically ill adult. AJR. 1985;144:901-905. Full Text @ EUR | Bibliographic Links | [Context Link]

5. Tocino IM, Westcott JL. Barotrauma. Radiol Clin North Am. 1996;34:59-81. Full Text @ EUR Bibliographic Links | [Context Link]

6. Heystraten FM. Pitfalls in medical imaging in thoracic injury. Injury. 1986;17:336. Full Text @ EUR Full Text | Bibliographic Links | [Context Link]

7. Groskin SA. Selected topics in chest trauma. Semin Ultrasound CT MR. 1996;17:119-141. Full Text

@ EUR | Full Text | Bibliographic Links | [Context Link] 


\section{IMAGE GALLERY}

Select All

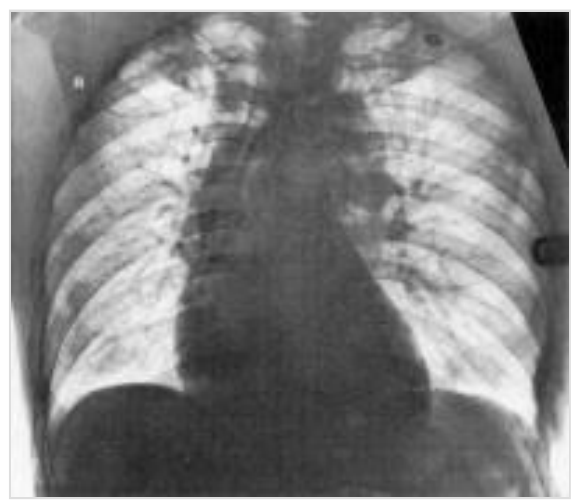

Figure 1

Back to Top

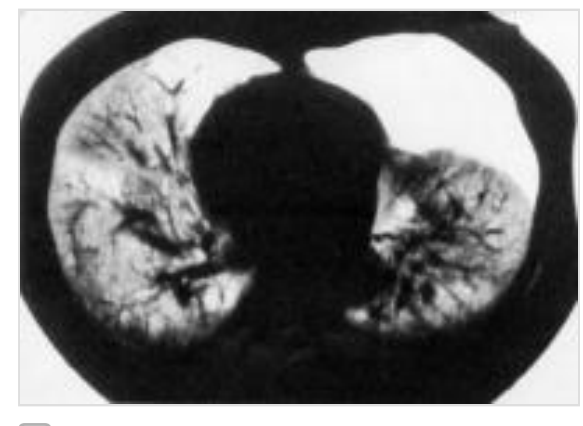

Figure 2

] Export Selected to PowerPoint

(c) 2016 Ovid Technologies, Inc. All rights reserved.

About Us | Contact Us | Terms of Use

OvidSP_UI03.20.00.112, SourceID 105955 